Medicaid-Daten der Jahre 2001 bis 2009 unter dem Klassiker signifikant weniger Klinikeinweisungen und Therapieabbrüche, allerdings um den Preis vermehrter Diabetes- und Hyperlipidämieinzidenz [4].

Versagt auch diese Substanz, könnte die weit verbreitete und gut verträgliche Augmentation mit Valproinsäure den Therapieerfolg verbessern [5].

Im US-amerikanisch-kanadischen Gemeinschaftsprojekt NAVIGATE (http://navigateconsultants.org) hat sich der Einfluss der Dauer einer unbehandelten Psychose (DUP) auf Lebensqualität und Psychopathologie ergeben. Patienten mit einer mittleren DUP von 74 Wochen hatten bei kürzerer DUP zu- sätzlich auch geringere Hospitalisierungsraten [6].

Ein Problem schizophrener Patienten ist die vergleichsweise geringe körperliche Aktivität. Nachdem sich durch eine Vielzahl von Studien in einer Metaanalyse ein Vorteil „mäßiger körperlicher Aktivität“ bestätigt hat [7], folgten jetzt „sportliche“ Empfehlungen von $150 \mathrm{Mi}$ nuten moderater oder 75 Minuten intensiver körperlicher Aktivität, ergänzt durch Krafttraining mindestens zweimal pro Woche [8]. Kein Nutzen im Hinblick auf die Negativsymptomatik bei Schizophrenie hat nach einer placebokontrollierten Studie indes die bei depressiven Erkrankungen sinnvolle, repetitive transkranielle Magnetstimulation
(rTMS) über dem dorsolateralen präfrontalen Kortex [9]. Dr. Andreas Häckel

Schizophrenien und Psychosen (Prof. Werner Strik). Psychiatrie Update, Mainz, 25.3.2017

1. Chong $\mathrm{HY}$ et al. Neuropsychiatric Disease and Treatment 2016; 12: 357-73

2. Siskind DJ et al. PloS One 2016; 11: (6)

3. Savitz AJ et al. Int J Neuropsychopharmacol 2016; 19 (7)

4. Stroup TS et al., Am J Psychiatry 2016; 173 (2): $166-73$

5. Tseng PT et al. Medicine 2016; 95 (4)

6. Kane JM et al., Am J Psychiatr2016; 173 (4): $362-72$

7. Dauwan $\mathrm{M}$ et al., Schizophrenia Bulletin 2016; 42 (3): 588-99

8. Rosenbaum $S$ et al., Early Intervention in Psychiatry 2016; 10: 435 -40

9. Hasan A et al., Schizophrenia Bulletin 2016; 42 (3): $608-18$

\section{Angsterkrankungen - hat das Mikrobiom hier seine Finger im Spiel?}

\section{Angst ist die häufigste neuropsychiatrische Erkrankung, aber auch eine Vorläufererkrankung für weitere psychiatrische Erkrankungen wie Depression oder Demenz. Eine wirksame Therapie der Angst ist teuer.}

A uch wenn der finanzielle Aufwand für eine wirksame Therapie der Angst hoch ist, schlägt der pharmakologische Anteil daran mit zirka $150 €$ pro Jahr kaum zu Buche. Gleichwohl ist aufgrund der protektiven Folgeeffekte eine Rendite der erfolgreichen Angsttherapie von Faktor 4 der eingesetzten Ressourcen zu erwarten [1], betonte Professor Katharina Domschke von der Universität Freiburg. So zeigte eine dänische Kohortenstudie bei über 3,3 Millionen Personen, dass bei Angsterkrankungen das Risiko einer nachfolgenden depressiven Episode dreifach und das einer rezidivierenden depressiven Störung im Vergleich zur Normalbevölkerung fünffach erhöht war. Weiter scheint durch eine Angsterkrankung die Konversion eines MCI (mild cognitive impairment) in eine manifeste Alzheimer-Demenz abhängig von der Ausprägung der Angstsymptomatik um $33-135 \%$ erhöht zu sein [2].

Auch bei der Angsterkrankung ist eine epigenetisch verringerte Methylierung bestimmter Gene als Risikofaktor beteiligt. Damit bildet die Epigenetik ein Scharnier zwischen Genen und Umwelt, sagte Domschke. Dies wurde etwa für das Monoaminoxidase A-Gen (MAOA) [3], aber auch für das FK506-binding protein-5-Gen (FKBP5) nachgewiesen [4]. Zugleich zeigte sich, dass sich diese reduzierte Methylierung im MAOAGen nach einer erfolgreichen kognitiven sechswöchigen Verhaltenstherapie wieder normalisierte, was quasi als molekularer Wirkmechanismus von Psychotherapie gesehen werden kann.

Auch das Mikrobiom spielt eine Rolle bei Angsterkrankungen. In einer Gruppe von über 10.000 Probanden erhöhte bereits eine einmalige antibiotische $\mathrm{Be}$ handlung mit Penicillin, Chinolonen, Sulfonamiden, Tetrazyklinen oder Imidazolen das Risiko einer späteren Angsterkrankung um das 1,17-1,44-fache [5]. Auf dieser Achse lässt sich aber offenbar auch therapeutisch ansetzen. In einer Pilotstudie mit 45 Probanden führte eine dreiwöchige präbiotische Diät (u. a. mit Galacto-Oligosacchariden) im Vergleich zur Kontrollgruppe unter Maltodextrin zu einer signifikanten Reduktion des morgendlichen Cortisolspiegels als Maß für den Stresslevel [6].

Entsprechend den S3-Leitlinien für Angsterkrankungen sollte Angstpatienten grundsätzlich Psycho- und Pharmakotherapie angeboten werden [7], erinnerte Domschke. SSRI und SNRI (selektive Serotonin/Serotonin-NoradrenalinWiederaufnahmehemmer) sind initial in halbierter Dosis langsam aufzudosieren („start low, raise slow“). Zudem sollte der Arzt die Therapie nach einer Remission noch für mindesten sechs bis zwölf Monate fortsetzen. Neu im Portfolio und offenbar gut wirksam bei generalisierter Angststöruntg sowie gemischter Angst und Depression ist auch ein Lavendelölextrakt [8].

Dr. Andreas Häckel

Angst (Prof. Katharina Domschke). Psychiatrie Update, Mainz, 25.3.2017

1. Chisholm D et al. Lancet Psychiatry 2016; 3: 415-24

2. Mah L et al. Am J Geriatr Psychiatry 2015; 2 . $515-23$

3. Ziegler C et al. Transl Psychiatry 2016; 6: e773

4. Yehuda R et al. Biol Psychiatry 2016; 80: 372-80

5. Lurie I et al. J Clin Psychiatry 2015; 76: 1522-8

6. Schmidt K et al. Psychpharmacology (Berl) 2015; 232: 1793-1801

7. Bandelow $B$ et al. www.awmf.org/leitlinien. html

8. Kasper S et al. Eur Neuropsychopharmacol 2016; 26: $331-40$ 